OPEN ACCESS

Edited by:

Hans Ulrich Häring, Tübingen University

Hospital, Germany

Reviewed by:

Erwin Dieter Schleicher

University of Tübingen, Germany

Peter Mikael Nilsson,

Lund University, Sweden

*Correspondence:

Jincao Chen

chenjincao@hotmail.com

Yibin Yang

1594559173@qq.com

Xiaohui Wu

wuxiaohui1971@sina.com

tThese authors have contributed equally to this work

Specialty section: This article was submitted to

Clinical Diabetes,

a section of the journal

Frontiers in Endocrinology

Received: 07 May 2020

Accepted: 17 June 2020

Published: 14 July 2020

Citation:

Liu Z, Li J, Huang J, Guo L, Gao R,

Luo K, Zeng G, Zhang T, Yi M,

Huang $Y$, Chen J, Yang $Y$ and Wu $X$ (2020) Association Between Diabetes and COVID-19: A Retrospective

Observational Study With a Large

Sample of 1,880 Cases in Leishenshan Hospital, Wuhan

Front. Endocrinol. 11:478.

doi: 10.3389/fendo.2020.00478

\section{Association Between Diabetes and COVID-19: A Retrospective Observational Study With a Large Sample of 1,880 Cases in Leishenshan Hospital, Wuhan}

\author{
Zeming Liu ${ }^{1 \dagger}$, Jinpeng $\mathrm{Li}^{2 \dagger}$, Jianglong Huang ${ }^{1 \dagger}$, Liang Guo ${ }^{1 \dagger}$, Rongfen Gao ${ }^{3 t}$, Kuan Luo ${ }^{4}$, \\ Guang Zeng ${ }^{5}$, Tingbao Zhang ${ }^{6}$, Meilin $\mathrm{Yi}^{7}$, Yihui Huang ${ }^{1}$, Jincao Chen ${ }^{6 *}$, Yibin Yang ${ }^{8 *}$ and \\ Xiaohui $W u^{6 *}$ \\ ${ }^{1}$ Department of Plastic Surgery, Zhongnan Hospital of Wuhan University, Wuhan, China, ${ }^{2}$ Department of Thyroid and Breast \\ Surgery, Zhongnan Hospital of Wuhan University, Wuhan, China, ${ }^{3}$ Department of Rheumatology and Immunology, Tongji \\ Medical College, Tongji Hospital, Huazhong University of Science and Technology, Wuhan, China, ${ }^{4}$ Department of \\ Neurosurgery, Wuhan Puren Hospital, Wuhan, China, ${ }^{5}$ Department of Urology, Zhongnan Hospital of Wuhan University, \\ Wuhan, China, ${ }^{6}$ Department of Neurosurgery, Zhongnan Hospital of Wuhan University, Wuhan, China, ${ }^{7}$ Department of Burn \\ and Plastic Surgery, College of Traditional Chinese Medicine, Three Gorges University, Yichang Hospital of Traditional \\ Chinese Medicine, Yichang, China, ${ }^{8}$ Department of Respiratory and Critical Care Medicine, Zhongnan Hospital of Wuhan \\ University, Wuhan, China
}

Aims: This study aimed to investigate the clinical courses and outcomes of diabetes mellitus patients with coronavirus disease 2019 (COVID-19) in Wuhan.

Methods: This study enrolled 1,880 consecutive patients with confirmed COVID-19 in Leishenshan Hospital. We collected and analyzed their data, including demographic data, history of comorbidity, clinical symptoms, laboratory tests, chest computed tomography (CT) images, treatment options, and survival.

Results: The percentages of patients with diabetes among the severe and critical COVID-19 cases were higher than those among the mild or general cases $(89.2 \%, 10.8$ vs. $0 \%, p=0.001$ ). However, patients with and without diabetes showed no difference in the follow-up period $(p=0.993)$. The mortality rate in patients with or without diabetes was $2.9 \%(n=4)$ and $1.1 \%(n=9)$, respectively $(p=0.114)$. Univariate and multivariate Cox regression analyses and the Kaplan-Meier curves did not show any statistically significant differences between patients with and without diabetes (all $p>0.05$ ).

Conclusions: Our study results suggested that diabetes had no effect on the prognosis of COVID-19 patients but had a negative association with their clinical courses. These results may be useful for clinicians in the management of diabetic patients with COVID-19.

Keywords: clinical courses, comorbidity, Coronavirus disease 2019 (COVID-19), diabetes mellitus, prognosis 


\section{INTRODUCTION}

Coronavirus disease 2019 (COVID-19) cause by severe acute respiratory syndrome-coronavirus 2 (SARS-CoV-2) was first identified in Wuhan, China, and has subsequently and quickly spread to more than 70 countries, including the United States, Spain, and Italy $(1,2)$. SARS-CoV-2 could cause severe, even lethal pneumonia and lung failure. As of April 25, 2020, more than 2,800,000 confirmed cases and 190,000 deaths of COVID-19 have been reported worldwide (3).

As an emergency specialty field hospital hosted by the Zhongnan hospital, Leishenshan Hospital was put into use in February to provide treatment for COVID-19 patients in Wuhan, marking a milestone in China's battle against COVID19. In Leishenshan Hospital, 1,880 patients were diagnosed with COVID-19 between February 8, 2020 and April 15, 2020. Among those patients, 139 cases had a previous diagnosis of diabetes mellitus.

Diabetes mellitus (DM) is a common endocrine disease (4) and widely known as a chronic, low-grade inflammatory disease caused by long-term immune system imbalance, metabolic syndrome, or nutrient excess $(5,6)$. Since the outbreak of COVID-19, several studies have been conducted to examine the relationship between diabetes and COVID-19 (1, 7, 8). However, their sample sizes were relatively small, and they did not clarify whether diabetes was a predictor of poor clinical outcomes and higher mortality of COVID-19. In this study, we investigated the association between diabetes as a comorbidity and negative clinical courses and outcomes of COVID-19 in a large sample of patients from a single hospital in Wuhan, China. The findings are expected to inform follow-up clinical treatment for patients with both diseases and COVID-19.

\section{MATERIALS AND METHODS}

\section{Study Design and Participants}

This single-center retrospective observational study was conducted on 1,880 patients diagnosed with COVID-19 at Leishenshan Hospital. After excluding cases without information about the history of diabetes, the final sample included 934 patients. Data were collected on admission or during hospitalization by attending physicians and documented in the form of electronic medical records. This study was approved by the Ethics Commission of the Zhongnan Hospital of Wuhan University (Approval number: 2020074) and carried out in accordance with the principles of the Declaration of Helsinki revised in 2008. The need for informed consent from patients was waived due to the time constraints during the COVID-19 emergency.

\section{Data Collection}

We collected data of 1,880 patients from their medical records. These data included age, sex, comorbidities, levels of illness severity (mild, moderate, severe, and critical), signs and symptoms, treatment options (i.e., antiviral therapy, antibiotic therapy, traditional Chinese medicine therapy, anticoagulation therapy, and administration of corticosteroid and Vitamin C), laboratory findings, and computed tomography (CT) images. All the data were reviewed and analyzed by two senior physicians.

\section{Outcomes}

The primary outcomes of this study included the survival of patients (alive vs. dead) and their highest level of illness severity. Another important primary outcome was the patients' CT score-a semi-quantitative scoring system-generated according to the features of CT images. Each of the CT image features, namely ground-glass opacities, reticulation or cords change, consolidation, and pleural effusions, was assigned 1 point. Score 1 was the sum of points, and score 2 ranged from 0 to 4 points based on the area of lung involvement: no involvement, $0 ;<25 \%$ involvement, $1 ; 26-50 \%$ involvement, 2 ; 51-75\% involvement, 3 ; and $76-100 \%$ involvement, 4 . The total score was the sum of score 1 and score 2 .

\section{Statistical Analysis}

Normally distributed continuous variables are expressed as means \pm standard deviations, and non-normally distributed continuous variables as medians and interquartile ranges. Meanwhile, categorical variables are described as frequencies and percentages. The independent $t$-test or Mann-Whitney $U$-test was conducted to compare continuous variables between the group of patients with diabetes (Group 2) and that of patients without diabetes (Group 1). Meanwhile, the $\chi^{2}$-test or Fisher's exact test was used to analyze the associations between categorical variables. Cox regression analysis and the Kaplan-Meier survival curves were performed to explore the prognosis of patients with and without diabetes. Besides, curve fitting analysis was conducted to further evaluate the association between the CT score and the duration from symptom onset (in days) in both groups. Two-sided $p<0.05$ were considered as statistically significant, and all statistical analyses were performed using SPSS (version 23.0 for windows).

\section{RESULTS}

\section{Study Participants' Demographic and Clinical Information and Laboratory Findings}

Table 1 shows that Group 2 consisted of 139 patients whereas Group 1 had 795 patients. The two groups showed no differences in gender distribution $(p=0.076)$, means of age $(p=0.773)$, and the number of patients with malignancy, nervous system disease, and digestive system disease (all $p>0.05$ ). However, they showed a significant difference in the number of patients with cardiovascular or pulmonary disease (both $p<0.05$ ). The percentages of patients with diabetes among the severe and critical COVID-19 cases were higher than among the mild or general cases $(89.2 \%, 10.8$ vs. $0 \%, p=0.001)$. However, no difference was observed in the follow-up period between patients with and without diabetes $(p=0.993)$. The mortality rate was $2.9 \%(n=4)$ in Group 2 and $1.1 \%(n=9)$ in Group $1(p=0.114)$. As shown in Table 2, the positive rate of IgG against SARS-CoV2 among patients with diabetes was lower than that among those without diabetes ( 82.5 vs. $91.8 \%, p=0.029$ ). 
TABLE 1 | Demographics and clinical characteristics of patients with COVID-19.

\begin{tabular}{|c|c|c|c|}
\hline Covariate & $\begin{array}{l}\text { Group 1* } \\
(n=795)\end{array}$ & $\begin{array}{l}\text { Group 2\# } \\
(n=139)\end{array}$ & $P$-value \\
\hline Age, year & $61.6 \pm 14.5$ & $64.5 \pm 10.0$ & 0.076 \\
\hline \multicolumn{4}{|l|}{ Sex } \\
\hline Male & 388 (48.8\%) & $66(47.5 \%)$ & 0.773 \\
\hline Female & 407 (51.2\%) & $73(52.5 \%)$ & \\
\hline \multicolumn{4}{|l|}{ Comorbidity } \\
\hline Cardiovascular disease & 275 (34.6\%) & 89 (64.0\%) & $<0.001$ \\
\hline Pulmonary disease & $90(11.8 \%)$ & $3(2.4 \%)$ & 0.001 \\
\hline Malignancy & $52(6.5 \%)$ & $14(10.1 \%)$ & 0.134 \\
\hline Nervous system disease & $47(5.9 \%)$ & $12(8.6 \%)$ & 0.224 \\
\hline Digestive system disease & $39(4.9 \%)$ & 7 (5.0\%) & 0.948 \\
\hline \multicolumn{4}{|l|}{ The highest level of severity } \\
\hline Mild and general & $19(2.4 \%)$ & $0(0)$ & 0.001 \\
\hline Severe & 739 (93.3\%) & $124(89.2 \%)$ & \\
\hline Critical & $34(4.3 \%)$ & $15(10.8 \%)$ & \\
\hline \multicolumn{4}{|l|}{ Status of illness when admission } \\
\hline Mild & $286(38.2 \%)$ & $43(30.9 \%)$ & 0.150 \\
\hline General & $211(28.2 \%)$ & $37(26.6 \%)$ & \\
\hline Severe & $235(31.4 \%)$ & $53(38.1 \%)$ & \\
\hline Critical & $17(2.3 \%)$ & $6(4.3 \%)$ & \\
\hline \multicolumn{4}{|l|}{ Symptoms when admitted to the hospital } \\
\hline Fever or Myalgia & 559 (78.8\%) & 95 (76.6\%) & 0.577 \\
\hline Respiratory system symptoms & $571(80.5 \%)$ & $104(83.9 \%)$ & 0.382 \\
\hline Digestive system symptoms & 77 (10.9\%) & $13(10.5 \%)$ & 0.901 \\
\hline Nervous system symptoms & $22(3.1 \%)$ & $5(4.0 \%)$ & 0.590 \\
\hline Antiviral therapy & $449(98.0 \%)$ & $86(97.7 \%)$ & 0.693 \\
\hline Antibiotic therapy & $297(98.3 \%)$ & 47 (97.9\%) & 0.590 \\
\hline The appliance of Vitamin C & $106(98.1 \%)$ & $19(100.0 \%)$ & 1.000 \\
\hline Traditional Chinese medicine therapy & $687(99.7 \%)$ & $118(100.0 \%)$ & 1.000 \\
\hline Anticoagulation treatment & $98(12.3 \%)$ & $21(15.1 \%)$ & 0.364 \\
\hline Use of corticosteroid & $82(10.3 \%)$ & $16(11.5 \%)$ & 0.671 \\
\hline Deaths & $9(1.1 \%)$ & 4 (2.9\%) & 0.114 \\
\hline Follow-up days & $22.4 \pm 9.7$ & $22.4 \pm 9.3$ & 0.993 \\
\hline
\end{tabular}

*Group 1 for patients without diabetes.

\#Group 2 for patients with diabetes.

\section{Survival Analysis}

Figure 1 presents the distribution of the number of deaths by the level of illness severity in both groups. There were six critical COVID-19 cases in Group 2, and three cases in Group 1. Besides, no deaths were observed among the severe cases in Group 2 while there were six deaths in Group 1. As shown in Table 3, the difference between the prognosis of Group 2 and that of Group 1 was demonstrated using either the univariate Cox regression analysis $(p=0.124)$ or the multivariate analysis ( $p=0.256$, Table 3 ). Similarly, the Kaplan-Meier curves showed no difference between the two groups $(p=0.111$, in Supplemental Figure 1).

\section{Evaluation of Chest CT Images}

Score 1 for all patients and that for patients without diabetes present the same tendency, i.e., rising and then descending (Figures 2A-C for all patients vs. Figures 2D-F for patients without diabetes). Similarly, score 1 for patients with diabetes sharply increased and significantly decreased after peaking at 2.70 points on day 23 (Figure 2G). However, score 2 for patients with diabetes showed an inverse tendency (Figure $2 \mathbf{H}$ ), reaching its nadir of 2.30 points on day 35. This inconsistent tendency might be because the largest area of lung involvement for patients with diabetes appeared earlier than that for all patients or those without diabetes. In patients with diabetes, the total score peaked at 5.30 points after 19 days (Figure 2I), exceeding the peak of 4.95 points on day 20 in all patients (Figure 2C) and that of 4.95 points on day 19 in patients without diabetes (Figure 2F). Curve fitting equation for Figure 2 and coefficients of each curve are shown in Supplemental Table 1.

\section{DISCUSSION}

The prevalence of diabetes mellitus is anticipated to increase substantially during the next decades worldwide and considered to be main cause of human deaths (9). People with diabetes are more susceptible to certain infectious diseases, such as staphylococcus aureus and mycobacterium tuberculosis, possibly because of their dysregulated immune system $(10,11)$. During the outbreak of SARS in 2003 in Guangzhou, Yang et al. reported that plasma glucose levels and diabetes were independent predictors for mortality and morbidity, and metabolic control might improve the prognosis in patients with SARS (12). Recently, COVID-19 has been a focal topic of research, and several investigations have focused on diabetes as a predictor of clinical course and prognosis of COVID-19 cases (1316). Certain studies found that diabetes negatively affected medical complications, including mortality, in COVID-19 cases $(13,17,18)$.

In our study, we collected data with a large sample from Leishenshan Hospital. We found that COVID-19 patients with DM were older and the proportion of patients with cardiovascular disease were higher among COVID-19 patients with DM than COVID-19 patients without DM. However, the latter had a higher proportion of pulmonary disease as comorbidities than the former. Furthermore, aspartate aminotransferase (AST) and platelet counts were lower in COVID-19 patients with diabetes than those without disease. In addition, non-diabetes-related comorbidities did not ameliorate the severity of illness on admission or improve survival outcomes, compared to diabetes-related ones. However, severe or critical COVID-19 cases were more prevalent among patients with diabetes than those did not suffer from this disease. A systematic review by Huang et al. revealed that diabetes was associated with mortality, severity, acute respiratory distress syndrome, and disease progression in patients with COVID19 (19). The results of our present study differ from those of previous ones possibly because Leishenshan Hospital is a designated hospital for COVID-19, and the standard of care for patients with diabetes has become better. Further researches are needed in the future to generate more precise results.

Having assessed the association between diabetes with the severity of COVID-19, Wu et al. found out the proportion of diabetes as a comorbidity among severe COVID-19 cases was 
TABLE 2 | Laboratory results in the COVID-19 patients with or without diabetes.

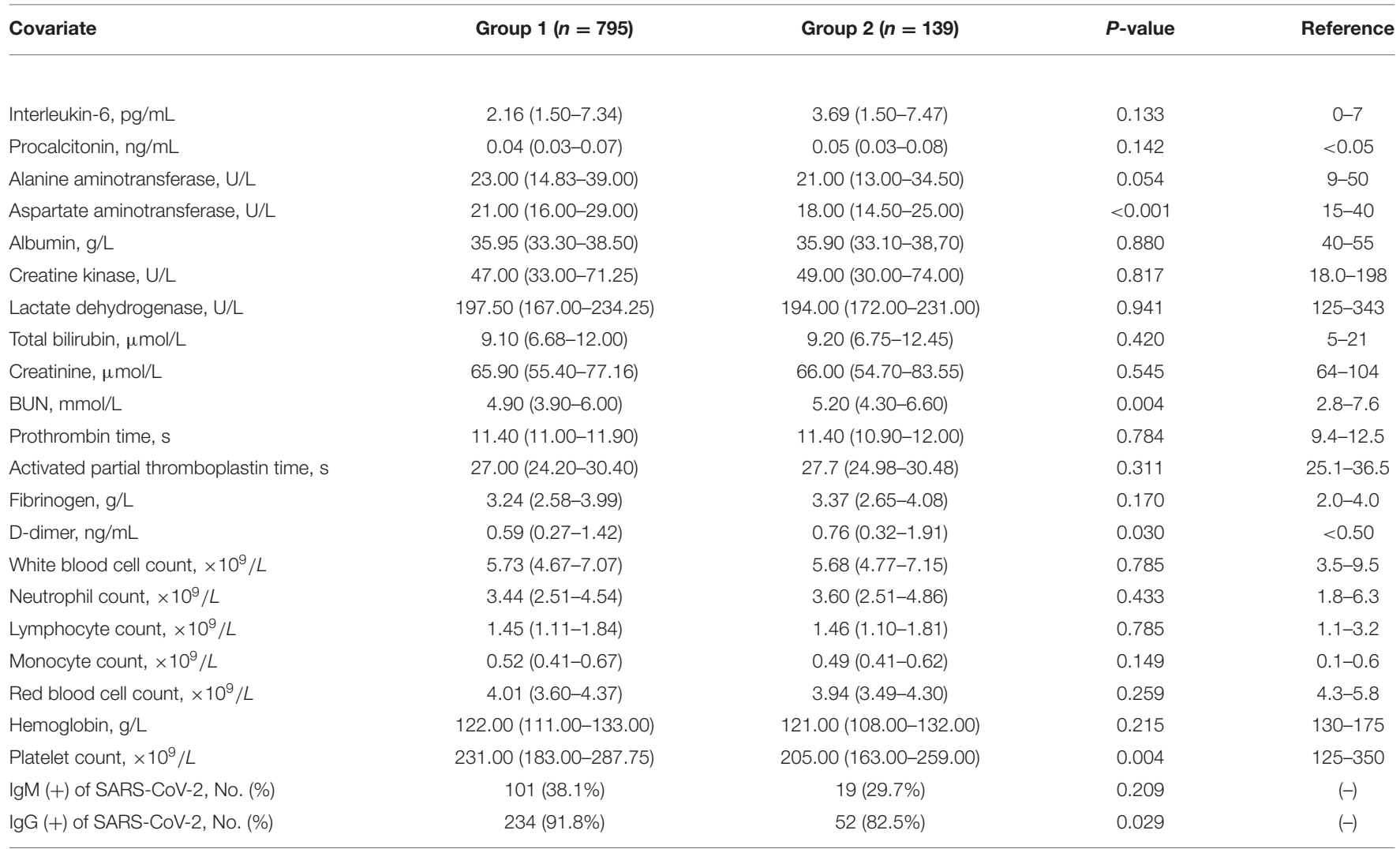

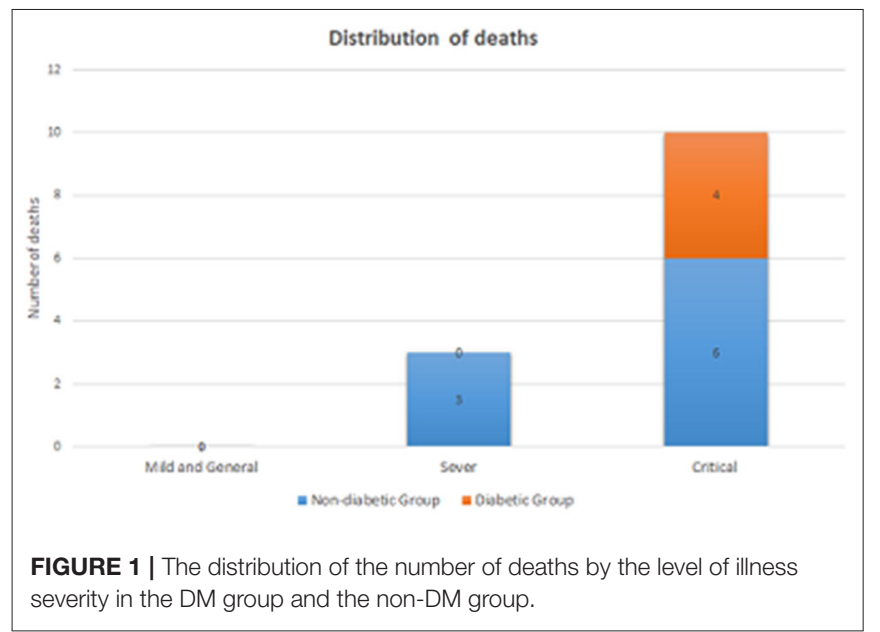

significantly higher than that among mild cases (14). Similarly, our data supported that the proportion of severe or critical COVID-19 cases among patients with diabetes was higher than that among those without diabetes. This means the clinical course of COVID-19 in patients with diabetes may be more severe than that in those without diabetes. Currently, the mechanisms behind this phenomenon remain unknown; however, high glucose levels may play a certain role in the impaired antibacterial neutrophil
TABLE 3 | Univariate and multivariate Cox regression analyses of diabetes for the mortality of patients with COVID-19.

\begin{tabular}{lccccc}
\hline Covariate & Group & \multicolumn{4}{c}{ Cox regression analysis } \\
\cline { 3 - 6 } & & HR & $95 \%$ Cl & P-value \\
\hline Univariate analysis & Group 1 & ref & & & \\
& Group 2 & 2.522 & 0.777 & 8.191 & 0.124 \\
Multivariate analysis* & Group 1 & ref & & & \\
& Group 2 & 2.135 & 0.576 & 7.912 & 0.256 \\
\hline
\end{tabular}

*Adjust for age, the history of cardiovascular disease, D-dimer, WBC, and lymphocyte count.

function and complications caused by chronic diabetes (9). In addition, the comorbidity with cardiovascular diseases, such as ischemic heart disease and heart failure, was reported to have an association with higher mortality due to COVID-19 (20). In our study population, $64.0 \%$ of patients with diabetes suffered from cardiovascular diseases, higher than that of those without diabetes. This may partly explain that the group of patients with diabetes had a higher proportion of COVID-19 cases in severe or critical conditions than the group of those without diabetes.

In our present study, biochemical laboratory test results showed that the increased AST level, an indicator of liver injury, was not observed in the blood of all patients, regardless of 


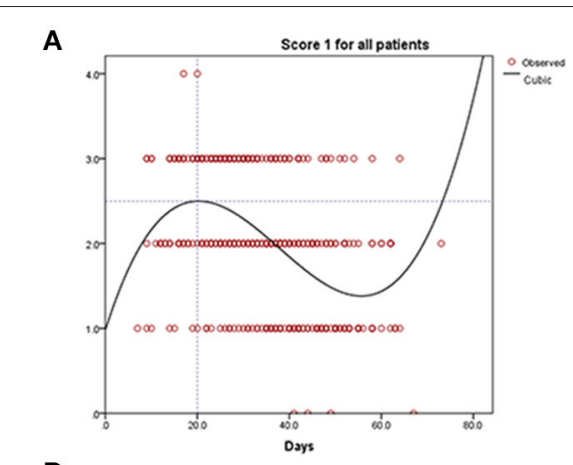

D

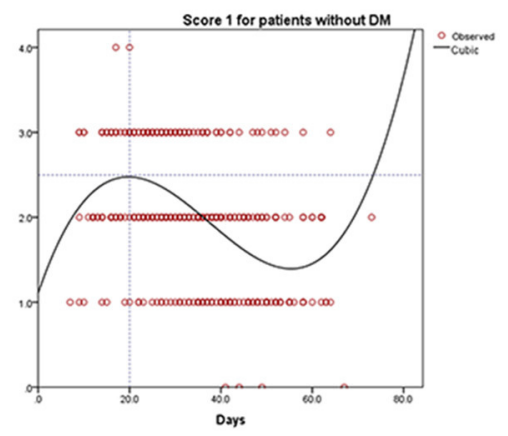

G

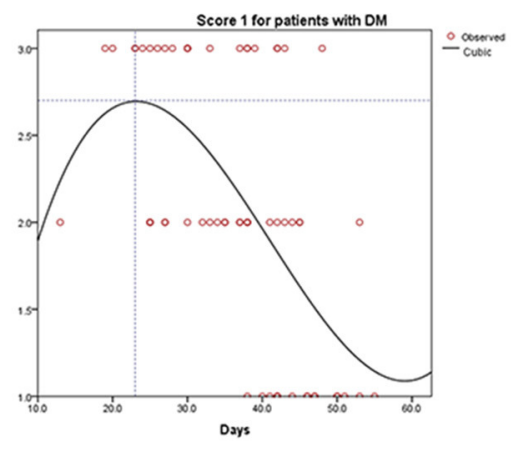

B

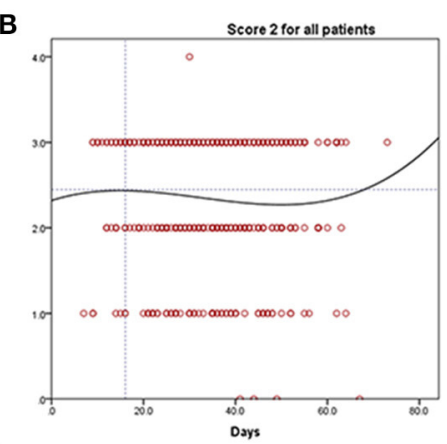

E

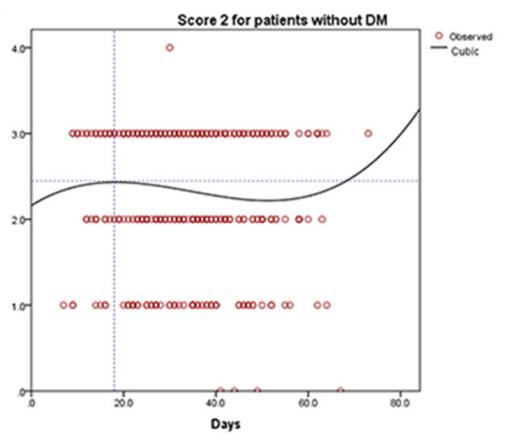

H

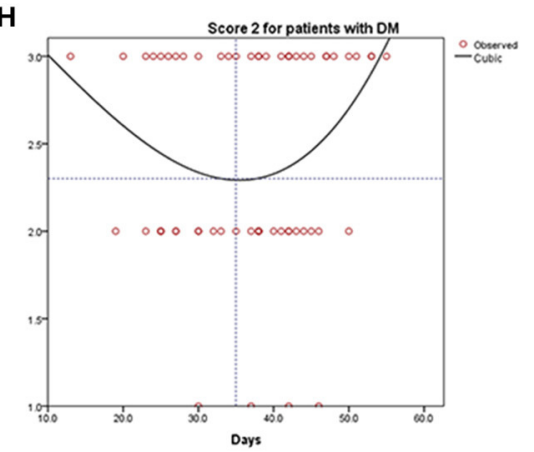

C

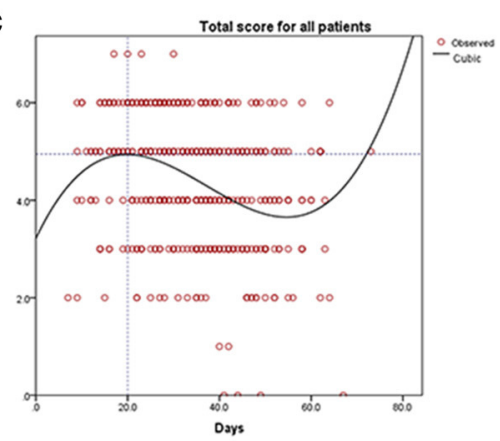

F

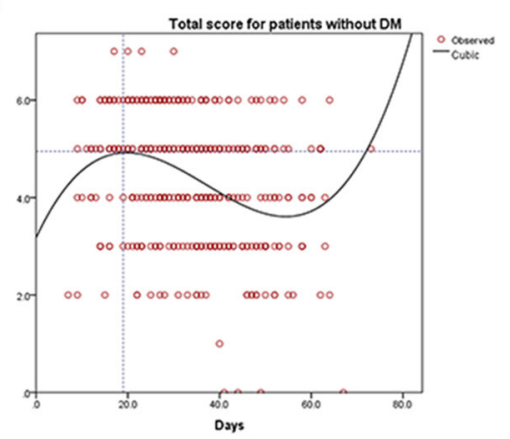

I

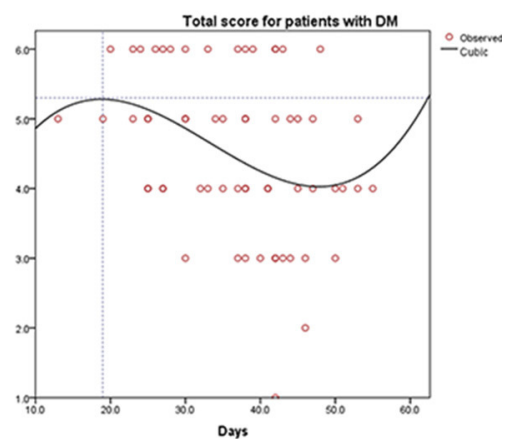

FIGURE 2 | Curve fitting analysis for all the COVID-19 patients (A-C), patients without diabetes mellitus (D-F), and patients with diabetes mellitus (G-I).

their status of diabetes. Furthermore, the two groups showed no difference in the levels of albumin and hemoglobin, suggesting that both groups had similar nutritional status. Having analyzed 138 COVID-19 patients, Wang et al. found that cytokine storms, sustained inflammatory response, and acute kidney injury might be associated with the mortality of COVID-19 patients (21). Cytokine storms also proved to be the main cause of eventual deaths among many patients with Ebola virus infections (13). In our study, however, patients with diabetes shared similar lymphocyte and neutrophil counts than patients without diabetes, the inflammation-related biomarkers (e.g., IL-6). These results indicate that the inflammatory response of patients with diabetes was not different from that of those without diabetes, resulting in similar rates of mortality due to COVID-19.

The use of angiotensin-converting enzyme 2 (ACE2) inhibitors and Angiotensin II Receptor Antagonists (ARBs) for COVID-19 patients has been of great controversy. ACE2 is the surface receptor for SARS-CoV-2 that directly interacts with the spike glycoprotein (22). Wrapp et al. has recently suggested that the affinity between ACE2 and the receptor binding domain of SARS-CoV-2 is much higher than that between ACE2 and the RBC of SARS-CoV (23). The expression of ACE2 in various organs, including the cardiovascular system, lungs, kidneys, and brain might explain why some COVID-19 patients died of multiple organ failure $(24,25)$. $\mathrm{ACE}$ inhibitors and $\mathrm{ARBs}$ may play a protective role in the treatment of COVID-19 cases (26). However, Cure et al. demonstrated that SARS-CoV-2 could enter cells by attaching to ACE2 enzymes and then cause infection. Furthermore, ACE inhibitors enhanced the sympathetic activity via the central stimulation and then increased pulmonary capillary leaking, possibly resulting in the development of ARDS. The authors suggested that the morbidity and mortality of COVID-19 among patients with diabetes would be higher if they used ACE inhibitors and ARBs (27). Our primary findings showed that as 
a comorbidity, diabetes did not increase the risk of mortality but negatively regulated the clinical course of COVID-19. Therefore, the treatment for COVID-19 patients should be appropriately adjusted.

Our study has serval limitations that need to be addressed. First, the retrospective, non-randomized nature led to sample heterogeneity. Second, we were not able to collect and analyze the characteristic data of patients with diabetes, such as the type of diabetes, glucose level, HbAlc and treatment options for diabetes due to the time limitation; hence, we could not analyze the data on anti-DM treatment although they could affect the clinical course and treatment outcome of COVID-19. Third, we did not examine the relative mechanism behind the effect of diabetes on COVID-19 in this study. Finally, the difference in disease progress and prognosis between the COVID-19 patients with or without diabetes may change with a longer follow-up period.

\section{CONCLUSION}

Our findings which were contradictory to those of previous studies with large sample sizes, suggested that diabetes did not significantly impact the prognosis of COVID-19 patients but negatively affect their clinical course. This may be helpful for clinicians in managing COVID-19 patients with diabetes. However, future prospective studies with larger sample sizes should focus on examining whether patients with diabetes are more at risk of COVID-19.

\section{REFERENCES}

1. Wu C, Chen X, Cai Y, Xia J, Zhou X, Xu S, et al. Risk factors associated with acute respiratory distress syndrome and death in patients with coronavirus disease 2019 pneumonia in Wuhan, China. JAMA Intern Med. (2020) e200994. doi: 10.1001/jamainternmed.2020.0994. [Epub ahead of print].

2. Huang Y, Tu M, Wang S, Chen S, Zhou W, Chen D, et al. Clinical characteristics of laboratory confirmed positive cases of SARS-CoV-2 infection in Wuhan, China: a retrospective single center analysis. Travel Med Infect Dis. (2020) 101606. doi: 10.1016/j.tmaid.2020.101606. [Epub ahead of print].

3. Chen N, Zhou M, Dong X, Qu J, Gong F, Han Y, et al. Epidemiological and clinical characteristics of 99 cases of 2019 novel coronavirus pneumonia in Wuhan, China: a descriptive study. Lancet. (2020) 395:507-13. doi: 10.1016/S0140-6736(20)30211-7

4. Ingelfinger JR, Jarcho JA. Increase in the Incidence of Diabetes and Its Implications. N Engl J Med. (2017) 376:1473-4. doi: 10.1056/NEJMe1616575

5. Guzman-Flores JM, Lopez-Briones S. Cells of innate and adaptive immunity in type 2 diabetes and obesity. Gac Med Mex. (2012) 148:381-9.

6. Shu CJ, Benoist C, Mathis D. The immune system's involvement in obesity-driven type 2 diabetes. Semin Immunol. (2012) 24:43642. doi: 10.1016/j.smim.2012.12.001

7. Bhatraju PK, Ghassemieh BJ, Nichols M, Kim R, Jerome KR, Nalla AK, et al. Covid-19 in critically ill patients in the seattle region - case series. $N$ Engl J Med. (2020) 382:2012-22. doi: 10.1056/NEJMoa2004500

8. Roncon L, Zuin M, Rigatelli G, Zuliani G. Diabetic patients with COVID-19 infection are at higher risk of ICU admission and poor short-term outcome. $J$ Clin Virol. (2020) 127:104354. doi: 10.1016/j.jcv.2020.104354

9. Knapp S. Diabetes and infection: is there a link?-a mini-review. Gerontology. (2013) 59:99-104. doi: 10.1159/000345107

\section{DATA AVAILABILITY STATEMENT}

The raw data supporting the conclusions of this article will be made available by the authors, without undue reservation, to any qualified researcher.

\section{ETHICS STATEMENT}

The studies involving human participants were reviewed and approved by Ethics Commission of the Zhongnan Hospital of Wuhan University Zhongnan Hospital of Wuhan University. The Ethics Committee waived the requirement of written informed consent for participation.

\section{AUTHOR CONTRIBUTIONS}

ZL: conceptualization, writing-review, and editing. JH: formal analysis and writing-original draft preparation. JL, LG, and RG: investigation and data curation. KL and GZ: methodology and software. TZ, MY, and YH: software, visualization, and validation. JC, YY, and XW: supervision and project administration. All authors: contributed to the article and approved the submitted version.

\section{SUPPLEMENTARY MATERIAL}

The Supplementary Material for this article can be found online at: https://www.frontiersin.org/articles/10.3389/fendo. 2020.00478/full\#supplementary-material

10. Muller LM, Gorter KJ, Hak E, Goudzwaard WL, Schellevis FG, Hoepelman $\mathrm{AI}$, et al. Increased risk of common infections in patients with type 1 and type 2 diabetes mellitus. Clin Infect Dis. (2005) 41:281-8. doi: 10.1086/431587

11. Hodgson K, Morris J, Bridson T, Govan B, Rush C, Ketheesan N. Immunological mechanisms contributing to the double burden of diabetes and intracellular bacterial infections. Immunology. (2015) 144:171-85. doi: 10.1111/imm.12394

12. Yang JK, Feng Y, Yuan MY, Yuan SY, Fu HJ, Wu BY, et al. Plasma glucose levels and diabetes are independent predictors for mortality and morbidity in patients with SARS. Diabet Med. (2006) 23:6238. doi: 10.1111/j.1464-5491.2006.01861.x

13. Guo W, Li M, Dong Y, Zhou H, Zhang Z, Tian C, et al. Diabetes is a risk factor for the progression and prognosis of COVID-19. Diabetes Metab Res Rev. (2020) e3319. doi: 10.1002/dmrr.3319. [Epub ahead of print].

14. Wu J, Li W, Shi X, Chen Z, Jiang B, Liu J, et al. Early antiviral treatment contributes to alleviate the severity and improve the prognosis of patients with novel coronavirus disease (COVID-19). J Intern Med. (2020). doi: 10.1111/joim.13063. [Epub ahead of print].

15. John TJ, John K. What is the role of angiotensin-converting enzyme 2 (ACE2) in COVID-19 infection in hypertensive patients with diabetes? Can J Cardiol. (2020) 36:969.e3. doi: 10.1016/j.cjca.2020.03.049

16. Gentile S, Strollo F, Ceriello A. COVID-19 infection in Italian people with diabetes: lessons learned for our future (an experience to be used). Diabetes Res Clin Pract. (2020) 162:108137. doi: 10.1016/j.diabres.2020. 108137

17. Hill MA, Mantzoros C, Sowers JR. Commentary: COVID-19 in patients with diabetes. Metabolism. (2020) 107:154217. doi: 10.1016/j.metabol.2020.154217

18. Klonoff DC, Umpierrez GE. COVID-19 in patients with diabetes: risk factors that increase morbidity. Metabolism. (2020). doi: 10.1016/j.metabol.2020.154224. [Epub ahead of print]. 
19. Huang I, Lim MA, Pranata R. Diabetes mellitus is associated with increased mortality and severity of disease in COVID-19 pneumonia - a systematic review, meta-analysis, and meta-regression. Diabetes Metab Syndr. (2020) 14:395-403. doi: 10.1016/j.dsx.2020.04.018

20. Zhou F, Yu T, Du R, Fan G, Liu Y, Liu Z, et al. Clinical course and risk factors for mortality of adult inpatients with COVID-19 in Wuhan, China: a retrospective cohort study. Lancet. (2020) 395:105462. doi: 10.1016/S0140-6736(20)30566-3

21. Wang D, Hu B, Hu C, Zhu F, Liu X, Zhang J, et al. Clinical characteristics of 138 hospitalized patients with 2019 novel coronavirus-infected pneumonia in Wuhan, China. JAMA. (2020). doi: 10.1001/jama.2020.1585. [Epub ahead of print].

22. Kuba K, Imai Y, Rao S, Gao H, Guo F, Guan B, et al. A crucial role of angiotensin converting enzyme 2 (ACE2) in SARS coronavirus-induced lung injury. Nat Med. (2005) 11:875-9. doi: 10.1038/nm1267

23. Wrapp D, Wang N, Corbett KS, Goldsmith JA, Hsieh CL, Abiona O, et al. Cryo-EM structure of the 2019-nCoV spike in the prefusion conformation. Science. (2020) 367:1260-3. doi: 10.1126/science.abb2507

24. Patel VB, Zhong JC, Grant MB, Oudit GY. Role of the ACE2/angiotensin 1-7 axis of the renin-angiotensin system in heart failure. Circ Res. (2016) 118:1313-26. doi: 10.1161/CIRCRESAHA.116.307708

25. Tipnis SR, Hooper NM, Hyde R, Karran E, Christie G, Turner AJ. A human homolog of angiotensin-converting enzyme. Cloning and functional expression as a captopril-insensitive carboxypeptidase. J Biol Chem. (2000) 275:33238-43. doi: 10.1074/jbc.M002615200

26. Kuster GM, Pfister O, Burkard T, Zhou Q, Twerenbold R, Haaf P, et al. SARS-CoV2: should inhibitors of the renin-angiotensin system be withdrawn in patients with COVID-19? Eur Heart J. (2020) 41:1801-3. doi: 10.1093/eurheartj/ehaa235

27. Cure E, Cumhur Cure M. Angiotensin-converting enzyme inhibitors and angiotensin receptor blockers may be harmful in patients with diabetes during COVID-19 pandemic. Diabetes Metab Syndr. (2020) 14:349-50. doi: 10.1016/j.dsx.2020. 04.019

Conflict of Interest: The authors declare that the research was conducted in the absence of any commercial or financial relationships that could be construed as a potential conflict of interest.

Copyright (c) $2020 \mathrm{Liu}$, Li, Huang, Guo, Gao, Luo, Zeng, Zhang, Yi, Huang, Chen, Yang and Wu. This is an open-access article distributed under the terms of the Creative Commons Attribution License (CC BY). The use, distribution or reproduction in other forums is permitted, provided the original author(s) and the copyright owner(s) are credited and that the original publication in this journal is cited, in accordance with accepted academic practice. No use, distribution or reproduction is permitted which does not comply with these terms. 